\section{Spin Polarized Electrons}

\section{H.C. Siegmann, Zürich}

(Lab. für Festkörperphysik, ETH)
The concept of spin polarized electron beams goes back to the discovery of the electron spin by Goudsmith and Uhlenbeck in 1925. There are two kinds of electron as soon as a direction is specified: those with spin up and those with spin down. If $n$ † (!) is the number of up (down) spin electrons, the degree $P$ of polarization along this direction is given by

$$
P=(n t-n !) /(n t+n !) \text {. }
$$

In 1956, Lee and Yang stimulated a general interest in the field by discovering that the newly born electrons emerging from a nucleus in $\beta$-decay were preferentially of one kind. However, the natural spin polarized electron beams from a radioactive source are so weak and electron-optically of such inferior quality that it is very hard to perform any experiments with them. At first, it seemed that free spin polarized electrons were an elusive rarity. Twenty five years later, it is obvious that this is not true at all. Spin polarization of electrons is quite hard to avoid, since it occurs whenever the spin orbit, exchange, or weak interaction comes into play.

\section{Producing a Beam of Spin Polarized Electrons}

For most purposes, it is best to start with the discovery of G. Lampel in 1967 that electrons excited across the bandgap of a semiconductor with circulary polarized light may be spin polarized. This is due to the selection rules in optical absorption. If right circularly polarized light is absorbed by any system, be it an atom or a crystal, the angular momentum of the system must change by $\hbar$ in the direction of the light beam. The highest occupied state in the semiconductor $\mathrm{GaAs}$ is an As-derived $4 p_{3 / 2}$-state, and the lowest unoccupied state a Ga-derived 4s-state. With circularly polarized light of an energy of $1.5 \mathrm{eV}$, one can induce transitions between these states that lead to an electron spin polarization of $50 \%$ at the bottom of the conduction bands. If the vacuum level is below the bottom of the conduction bands (a condition which is called negative electron affinity), the electrons can escape into vacuum, suitable electron optical elements. The photocurrent leaving the $\mathrm{GaAs}$ cathode depends on the intensity of the exciting radiation at $1.5 \mathrm{eV} ; 20 \mu \mathrm{A} / \mathrm{mW}$ are readily obtained.

The intensity of the electron beam is space charge limited just as in ordinary electron guns. However, the beam may be twice as monochromatic as a beam from a hot filament gun. A strong advantage occurs also in pulsed operation, since the exand an electron beam can be formed with citing radiation can be a pulsed laser. Although most researchers have reported a polarization of the electron beam ranging from 30 to $40 \%$, with Be-doped GaAs grown by molecular epitaxy, it is possible to reach the theoretical value of $50 \%$ in addition to a very good lifetime. The most important feature for the study of spin dependent interactions is the reversibility of $P$ without affecting other characteristics of the electron beam, by simply switching the light from right to left circularly polarized. With lock-in-techniques, one can then study spin dependent interactions down to a level of $10^{5}$. This feature made it possible, for instance, to prove at Stanford in 1978 that parity is violated in electron proton scattering.

\section{Measuring Spin Polarization of an Elec- tron Beam}

The spin polarization may be measured by differential scattering of electrons on heavy atoms. One determines the intensity of electrons scattered from a target at a given energy into two symmetrical solid angles to the right and to the left of the target. Owing to spin orbit coupling, the spin-up electrons are preferentially scattered to the right and the spin down to the left. The preferential direction, along which $P$ is measured, is the direction perpendicular to the scattering plane. However, the fraction of the incident electron beam that scatters into these solid angles is typically less than $10^{-3}$ and, even for a fully polarized electron beam, the normalized difference between the right and the left channel is $<0.3$. The poor efficiency of $<10^{-3}$ and the low analyzing power of $<0.3$ in addition to the high cost of angular- and energy selective electron counting techniques have hindered, thus far, a wide application and use of the spin degree of freedom in sience and technology. Nevertheless, much simpler and more effective schemes have started to come up recently: for instance, diffuse electron scattering on a polycrystalline target at convenient electron energies of $100 \mathrm{eV}$ provides a very efficient and a much simpler way of measuring $P$.

Suppose that the electron beam under investigation is at an energy of $\sim 100 \mathrm{eV}$ and hits a polycrystalline target, typically gold, at an angle of $\sim 45^{\circ}$ with the surface normal. At these low energies, most of the elastic electrons are scattered by the first atomic layer. The spin up electrons are preferentially scattered to the right, where we have vacuum, and consequently their intensity $i_{\mathrm{s}}$ can be detected by measuring the current flowing to a collector shell. The spin down electrons are preferentially scattered to the left, that is into the target, and it is very likely that they will loose energy by plasmon and electron hole pair production, and be absorbed. Hence their intensity can be measured by connecting a meter to the target and observing the absorbed current $i_{\mathrm{a}}$. However, there is a large spin independent background in both $i_{\mathrm{s}}$ and $i_{\mathrm{a}}$ from various causes, such as multiple scattering and production of secondary electrons.

The simplest way to eliminate completely this background is to let the electron beam impinge at an energy $E_{0}$ at which $i_{3}=0$ for a beam with $P=0$. $E_{0}$ is the energy at which the current of absorbed primaries is exactly compensated by the current of elastically and inelastically scattered primaries and secondaries leaving the sample. Two such energies are known to exist with target materials having a high yield of secondary electrons. This is the principle of a bridge circuit. Due to the spin dependence of the various scattering processes involved, the equilibrium of the bridge is offset when the beam is spin polarized. We then have $P=$ const $i_{a}\left(E_{0}\right)$, and one determines $P$ simply by measuring $i_{a}\left(E_{0}\right)$.

Another more general way to compensate the spin independent background of $i_{\mathrm{a}}$

\section{Universität Innsbruck}

Die Universitätsdirektion der Universität Innsbruck bittet um die Aufnahme nachstehender Stellenausschreibung:

"An der Universität Innsbruck ist die Planstelle eines

Ordentlichen Universitätsprofessors für Angewandte Physik zu besetzen. mit besonderer Berücksichtigung der Mikroelektronik

Bewerber sollen auf dem genannten Gebiet in Forschung und Lehre hervorragend qualifiziert sein und über reiche Erfahrung auf dem Gebiet der Entwicklung elektronischer Bauelemente und der Anwendung von Mikroprozessoren verfügen. Vom künftigen Ordinarius wird eine besondere Bereitschaft zur Zusammenarbeit mit anderen Institutionen und der Industrie, besonders in Tirol und Vorarlberg, erwartet.

Bewerber werden gebeten, ihre Unterlagen (Lebenslauf, Darstellung der bisherigen Tätigkeit in Forschung und Lehre, Schriftenverzeichnis und Beilage wichtiger Publikationen) bis 30 April 1983 beim Dekanat der Naturwissenschaftlichen Fakultät der Universität Innsbruck, Innrain 52, A-6020 Innsbruck, einzureichen.

Univ.-Prof. Dr. Johann GRUBER, Dekan" 


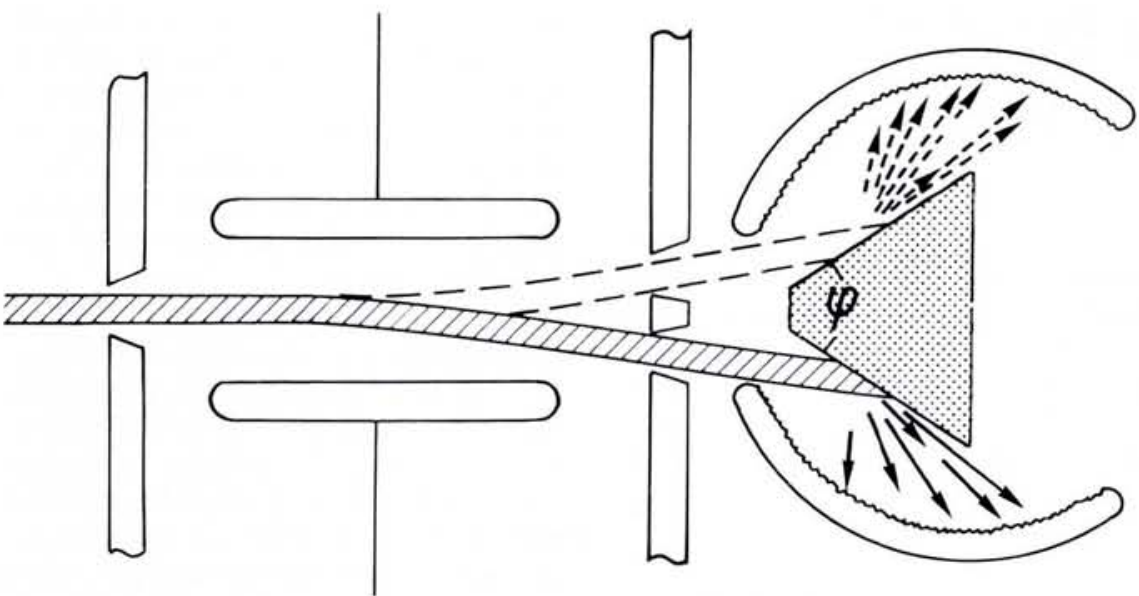

and/or $i_{\mathrm{s}}$ which functions at any energy of the electron beam is depicted in Fig. 1. The target is a prism or a truncated cone, and by applying a voltage to the condenser, the incident electron beam can be made to hit either the right or the left side of the prism. The spin independent part of $i_{a}$ does not change on switching the beam, whereas the spin dependent part changes in phase with the polarity of the voltage applied to the condenser as soon as $P \neq 0$. With an alternating polarity, $i_{\text {a }}$ will contain an AC component proportional to $P$, which may be extracted by lock-in-techniques with high accuracy. This absorbed current spin detector has an effective analyzing power of unity and, depending on the target material, an efficiency of up to $10^{-1}$ which it is expected will be further improved. It is also conceivable that other schemes based on detecting the luminescence produced by the impact of polarized electrons will prove to be useful in the future.

\section{Spin Selective Scattering on Electrons} on Magnetic Atoms

The electron-hydrogen is the most fundamental of all electron-atom collision problems. Mathematically, it requires a solution to a three body problem. Groups at Yale University and at Bielefeld ${ }^{1}$ ) scattered

Fig. 2 - The spin dependence $S$ of electrons scattered elastically from the ferromagnetic glass $\mathrm{Fe}_{40} \mathrm{Ni}_{40} \mathrm{~B}_{20}$ vs. electron energy. The spin polarized electron beam from a GaAs-gun was incident normal to the surface, and the intensity $i+(i-)$ of electrons scattered elastically into a cone centred at $14^{\circ}$ from the surface normal was measured for $P$ parallel (antiparallel) to the magnetization (from Ref. 2).

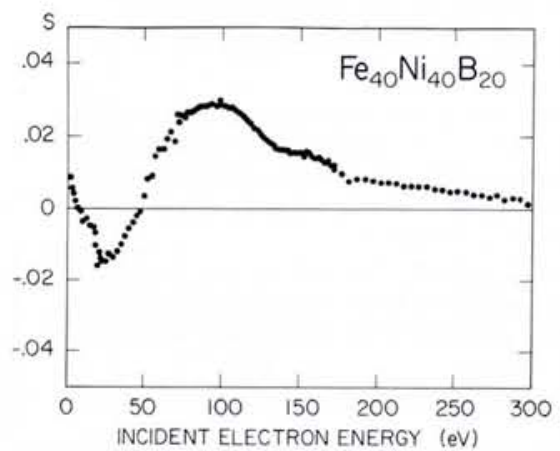

a spin polarized electron beam on a spin polarized hydrogen beam and their findings showed that even this simplest possible case is still hard to handle theoretically. If one now takes an atom of iron and asks the question how the scattering of an electron depends on the relative orientation of the atomic moment and the electron spin, one is prepared for surprises and difficulties. Yet this scattering is basic to the understanding of metallic magnetism and many other practical problems. D.T. Pierce and coworkers $^{2}$ ) have shown that one can at least handle it experimentally.

In practice, free $\mathrm{Fe}$ atoms are not the most interesting as they are quite different from the $\mathrm{Fe}$ cores carrying the magnetic moment in a metal. The problem amounts to finding a way to avoid the effects of diffraction and multiple scattering that normally play a dominant rôle in the scattering of electrons from atoms in crystalline solids. A further worry is the stray magnetic fields that are generated by the magnetization produced on aligning the $\mathrm{Fe}$ atoms.

Most objectives can be achieved by using as scatterer a ferromagnetic metallic glass which will have the structure of a liquid, i.e. the crystal diffraction will be negligible. Also, multiple scattering of electrons will contribute less than $30 \%$ to the intensity in the backward scattering direction. This arises because the singly backscattered electrons have the shortest path within the metal and hence are least attenuated by plasmon and electron-hole pair production. Finally, due to the lack of crystalline anisotropy in a liquid, the external field required to induce magnetic saturation is very low. This in turn leads to low magnetic stray fields outside the sample such that even very low energy electrons of $2 \mathrm{eV}$ are hardly deflected at all.

The intensity of electrons back-scattered by $i^{ \pm}=\lambda^{ \pm} \sigma^{ \pm}$, where $\lambda^{+}\left(\lambda^{-}\right)$is the mean free path and $\sigma^{+}\left(\sigma^{-}\right)$the "atomic scattering cross-section for an electron with spin parallel (antiparallel) to the majority spin direction in the solid. The spin dependence of the elastic scattering defined as $S=$ $\left(i^{+}-i^{-}\right) /\left(i^{+}+i^{-}\right)$is shown in Fig. 2 for a from a metallic ferromagnetic glass is given
Fig. 1 - A possible version of the absorbed cur rent spin detector. A meter is connected to the target hit by the electron beam under investiga tion which allows the absorbed current $i_{a}$ to be measured. The target is a prism, and by applying an $A C$ voltage to the condenser, the electron beam hits alternately the right and left side of the prism, thereby creating a change of sign of the spin dependent part of $i_{a}$. This AC fraction of $i_{a}$ is proportional to the spin polarization $P$ and may be extracted from the spin independent back ground with lock-in-techniques. An analogous technique may be applied to measure the spin dependent part of the diffusely scattered current $i_{s}$ reaching the collector shell encircling the target.

$\mathrm{Fe}_{40} \mathrm{Ni}_{40} \mathrm{~B}_{20}$ glass, for incident electron energies ranging from $2-300 \mathrm{eV}$. We see that $S$ is quite small, of the order of $10^{-2}$, yet this can easily be measured by using the spin modulated beam from a GaAs source. We also see interesting structure and changes of sign.

How can this be understood ? J.S. Helman, R. Feder, Sheng Wei Wang and very recently R.K. Nesbet have proposed theoretical models to understand the observations displayed in Fig. 2 and whereas it is not yet decided which approach is best, it is obvious already that such studies will greatly contribute to the understanding of magnetism in metals.

Meanwhile one can use spin selective electron scattering to investigate surface magnetism. $S$ is, in fact, a measure of the average surface magnetization $M_{s}^{*}$ in a sheet, of thickness corresponding to the probing depth of the electrons. For example, electrons which scatter in $\mathrm{Ni}$ at an energy of $90 \mathrm{eV}$ have a mean free path for inelastic scattering of $5 \AA$. Thus, the probing depth is $2.5 \AA$ corresponding to travel, on the average, of $1 / 2 \times 5 \AA$ into and out of the sample, which is about 1.5 atomic layers.

From symmetry we know that $S$ must be proportional to an odd power of $M_{\mathrm{s}}$, i.e.

$S=\alpha M_{\mathrm{s}}^{*}+\beta M_{\mathrm{s}^{*}}^{* 3}+\gamma M_{\mathrm{s}}^{* 5}+\ldots$

When there is only single scattering, we have $S=\alpha M_{s}^{*}$ at all temperatures. This applies to electrons back-scattered from $\mathrm{Ni}_{40} \mathrm{Fe}_{40} \mathrm{~B}_{20}$, and one can now study the temperature dependence of the surface magnetization. We see from Fig. 3, that the surface magnetization decreases about three times as fast, but with the same $T^{3 / 2}$ law as the bulk magnetization. This is due to special magnons excited at the surface and was predicted by D.C. Mills and A.A. Maradudin 15 years ago, but could never be unambiguously verified by conventional techniques as the disturbance is confined to the very first layers.

In a single crystal, the electrons are diffracted and multiply scattered, and it is not clear how $S$ is related to $M_{\text {s. }}$. However, as $\mathrm{M}_{\mathrm{s}}^{*} \rightarrow 0$ as $T \rightarrow T_{\mathrm{c}}$, the higher orders of $M$ can be neglected, and we have again $S=$ 


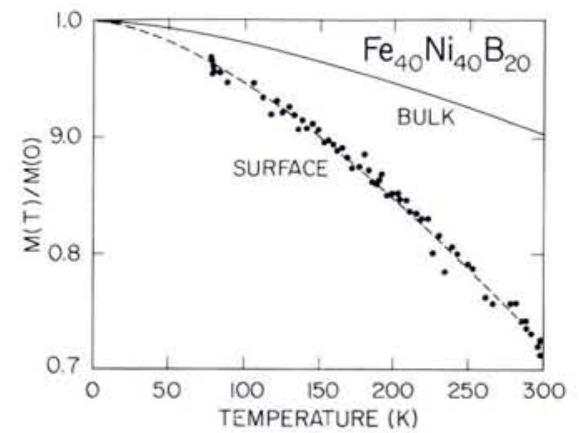

Fig. 3 - The temperature dependence of the bulk $\left(M_{B}\right)$ and surface $\left(M_{S}\right)$ magnetization with a ferromagnetic metallic glass (from Ref. 2). $M_{s}$ was obtained from the spin dependence $S$ of the elastic electron scattering at an energy of $90 \mathrm{eV}$. $M_{B}$ was measured with a moving sample magnetometer.

$\alpha M_{\mathrm{s}}$. As a result, magnetic single crystals have been used by S.F. Alvarado, M. Campagna, and $\mathrm{H}$. Hopster $^{3}$ ) to measure $M$ close to $T_{c}$ where short wavelength magnons prevail. Fig. 4 shows the results obtained with a (110) surface of $\mathrm{Ni}$ where the electron energy was chosen to be $49 \mathrm{eV}$ to ensure the highest possible surface sensitivity. We see that $M_{\mathrm{s}}^{*}$ obtained from spin selective electron scattering decreases much more slowly as we approach $T_{c}$ than the bulk magnetization as measured by scattering of neutrons.

This is the first study of the magnetic critical behaviour of a well characterized free surface. It was found that the surface magnetization decreases with the critical exponent $\beta_{1}=0.79 \pm 0.02$ for $0.002 \leq$ $T / T_{c} \leq 0.1$. Similar data for a $(100)$ surface indicate $\beta_{1}=0.81 \pm 0.02$. Experimental results on the critical behaviour of semiinfinite and true two-dimensional systems are a subject of great current interest, and will be essential to check on the numerous theoretical models.

Fig. 4 - The temperature dependence of the magnetization in Ni as determined by scattering of neutrons, and by the spin dependence $S$ of the elastic scattering of electrons at $49 \mathrm{eV}$ from a Ni (110) surface (by courtesy of Maurice Campagna).

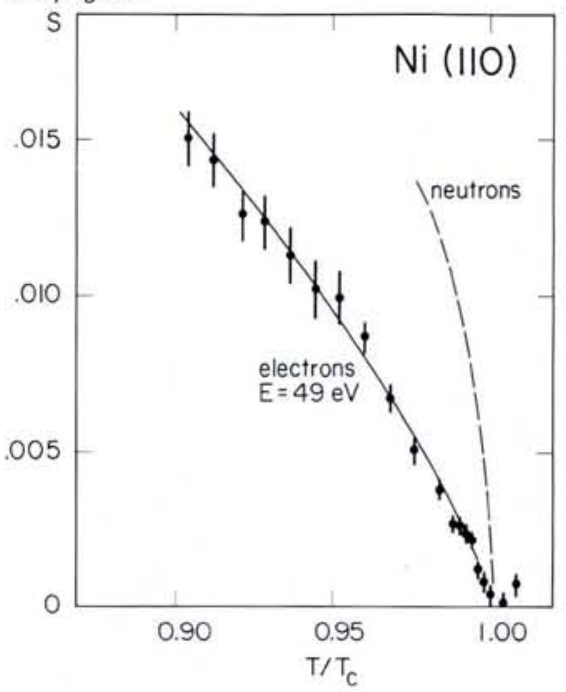

Experiments with Polarized Electrons

There are many more experiments that can be performed with polarized electrons. In the above example of spin selective electron scattering, a spin polarized electron source is needed, but no detector for spin polarization. In a second class of experiment, only the detector is needed, but no source. Great contributions to the understanding of electron states in atoms have been made through this type of experiment ${ }^{4}$ ) which includes photoemission of electrons from magnetized materials. Here the electrons are polarized in the solid by the exchange interaction and/or by an external magnetic field. In most cases, the spin is conserved in the process of photoemission, and hence the degree of spin polarization $P$ of the photoelectrons excited with monochromatic light and measured at various kinetic energies and angles of emission, yields a unique picture of the energy distribution of the magnetization. This is how the important many-body effects in understanding itinerant magnetism in $\mathrm{Ni}$ were quantitatively ascertained.

One of the unique features of photoemission is the possibility it offers of selecting the electron states that one wants to study, e.g. by choosing an appropriate photon energy. This led for instance, to the decomposition of the total magnetization into parts contributed by the $5 \mathrm{f}$ - and the $6 \mathrm{~d}$-states in the uranium compounds. Another example of an interesting application of spin polarized photoemission is surface magnetochemistry. Fig. 5 displays measurements on a chromium (100) surface ${ }^{5}$ ) with a very small amount of oxygen incorporated below the first layer. Such a surface is clearly magnetic with a transition temperature $T_{c}>T_{N}$, where $T_{N}$ is the Néel point of antiferromagnetic bulk chromium. If, however, the oxygen is completely removed or located on top of the (100)-surface, no spin polarized photoelectrons are emitted, indicating that the para-or antiferromagnetic state typical of bulk $\mathrm{Cr}$ prevails.

This has immediate practical applications: simply by measuring the magnetic suceptibility, one may now determine whether oxygen has been incorporated or has remained at the surface. Indeed, the magnetic properties of a surface are probably the finest sensors of its chemical state. The advent of new storage rings as dedicated sources for synchrotron radiation will provide further impetus for these and other types of spin polarized photoemission experiments.

Another example of the second type of experiment in which only the detector for spin polarization is needed, is the production of secondary electrons from a magnetized material by impact with primary unpolarized electrons $\left.{ }^{6}\right)$. As the primary electron beam may be focussed into a spot of diameter down to $100 \AA$, the spin polarization of the secondary electrons emitted

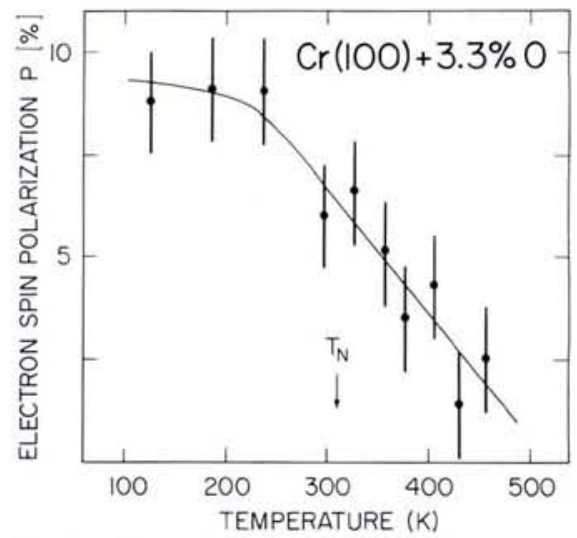

Fig. 5 - The spin polarization of photoelectons, emitted from a chromium (100) surface with a very small amount of subsurface oxygen, vs. temperature (from Ref. 5). The Néel point $T_{N}$ of bulk $\mathrm{Cr}$ is also indicated.

from the sample reflects the magnetization of that spot. This allows, for instance, observation of magnetic domains with an unprecedented spatial resolution. It may be used to study domain walls and switching of magnetic domains, or to measure surface magnetization curves. All of these are hot topics in the magnetic recording industry.

In a third class of experiment, one has both a spin polarized electron source and a detector for spin polarization. This of course yields the best information. It has been shown recently ${ }^{7}$ ) that it is possible to direct a primary beam of spin polarized electrons onto a metal, energy analyze the secondary electrons emitted at a well defined angle with respect to the primary beam, and still measure the spin polarization of the secondaries. With a non-magnetic material, spin polarization of the secondary

\section{UNIVERSITY OF MANCHESTER DEPARTMENT OF PHYSICS}

\section{Postdoctoral Research Associate in Experimental Nuclear Structure Physics}

Applications are invited for the above post, funded by SERC for a period of 3 years from 1 February, 1983. The successful candidate will be expected to pursue research at the Nuclear Structure Facility, a 20-30 MV tandem Van de Graaff which is now operational at the Daresbury Laboratory. Assistance with the commissioning and use of the magnetic spectrometer at the Daresbury Laboratory will also form a fraction of the candidate's duties. Applicants are expected to hold a Ph.D. degree and should have an aptitude for pursuing research in experimental physics. Salary range p.a. $£ 6,375-£ 7,655$ (Superannuation). Applications, with full C.V. and names of two referees to: Dr. R. Chapman, Department of Physics, The University, Manchester MI3 9PL, from whom further details may be obtained. 
electrons can arise only through the quantum-mechanical exchange in the electronelectron collision. Hence this experiment promises a unique experimental test of the exchange interaction of electrons in a solid, employing directly the symmetry properties of the fermions.

The necessity for a vacuum in the experiments with polarized electrons as described here may be overcome in the future, especially with experiments of the second type involving the emission of polarized electrons from solids. To measure $P$, the sample surface could be covered with a semiconductor, and the circular polarization of the recombination radiation of the injected electrons with appropriate states could be observed, reversing the principle of operation of the spin polarized electron source. Spin dependent tunnelling into a magnetic semiconductor or superconductor, or spin selective electron scattering in point contacts containing magnetic impurities have also been proposed, or used as solid state detectors for electron spin polarization. $\mathrm{P} / \mathrm{n}$ junctions are the basis of present electronics, and can be made with semiconductors only. However, a spin up/down junction is also conceivable with metals, which leads one rightly to suspect that this field is only at its very beginning.

\section{REFERENCES}

1. Fletcher G.D., Alguard M.J., Gay T.J., Hughes V.W., Tu C.W., Wainwright P.F., Lubell M.S., Raith W. and Tang F.C., Phys. Rev. Lett. 48 (1982) 1671.

2. Pierce D.T., Celotta R.J., Unguris J. and Siegmann H.C., Phys. Rev. B 26 (1982) 2566. 3. Alvarado S.F., Campagna M. and Hopster H., Phys. Rev. Lett. 48 (1982) 51 and Phys. Rev. Lett. 48 (1982) 1768

4. Schönhense G., Heinzmann U., Kessler J. and Cherepkov N.A., Phys. Rev. Lett. 48 (1982) 603 and refs. cited therein.

5. Meier F., Pescia D. and Schriber T., Phys. Rev. Lett. 48 (1982) 645.

6. Unguris J., Pierce D.T., Agalejs A. and Celotta R.J., Phys. Rev. Lett. 49 (1982) 72.

7. Ravano G., Erbudak M. and Siegmann H.C., Phys. Rev. Lett. 49 (1982) 80.

\section{International Facilities for Physics Research}

Preceding the meeting of the EPS Council which will take place in Copenhagen on 24/25 March 1983, a Symposium on International Facilities for Physics Research will be held from 21-23 March, partly in Copenhagen and partly at the Risø National Laboratory which is celebrating its 25 th anniversary at the same time.

The Symposium which is co-sponsored by the American Physical Society, Ris $\emptyset$ and the NOVO Foundation is a follow-up to the Symposium on the European Great Projects that took place in Rome on 26/27 March 1979 (see Europhysics News, 10 (1979) 4).

Principal topics covered by the Symposium will be:
1) Accelerators for nuclear physics and for high energy research;

2) Fusion research programmes;

3) Astrophysics in space and groundbased astronomical telescopes;

4) Neutron beam facilities;

5) Synchrotron radiation sources and facilities for condensed matter research.

Papers will be presented on both European and non-European programmes and the final session will be devoted to two panel discussions; one on new facilities for the physics community and the second on promoting international collaboration in physics.

\section{Hewlett-Packard Europhysics Prize}

The formal citation awarding the 1983 Hewlett-Packard Europhysics Prize to Professor Isaac Silvera, as announced last month, states that it is in recognition of his work on Atomic and Solid Hydrogen.

Readers of Europhysics News will already be familiar with some of the outstanding achievements of Professor Silvera. In May 1980 we published an article contributed by him in collaboration with J.T.M. Walraven describing the experiments at the University of Amsterdam on the "Stabilization of Monoatomic Hydrogen - a New Bose Gas", whereby polarized atomic hydrogen was condensed on to a film of liquid helium at a temperature of $270 \mathrm{mK}$. Then, in August/September 1982, Prof. Silvera summarized the lectures he had been giving around Europe in his capacity as EPS Travelling lecturer on "New Phases of Molecular and Atomic Hydrogen under Extreme Conditions". This reviewed experiments on the behaviour of solid hydrogen at pressures going up to over $0.5 \mathrm{Mbar}$. Of particular interest was the detection of the broken (rotational) symmetry phase transition in $D_{2}$ at $1 \mathrm{~K}$, at a pressure of $278 \mathrm{kbar}$ - a value much higher than predicted. He also gave the most recent news of polarized atomic hydrogen gas being stabilized at a density of $3 \times 10^{17}$ atom $/ \mathrm{cm}^{3}$.

The 1983 prize will be presented on the second day of the General Conference of the EPS Condensed Matter Division to be held in Lausanne from 28-30 March 1983. Professor Silvera will be one of the speakers at the Conference.

Donated annually by the HewlettPackard Co., the prize is given for outstanding achievement in the field of solid state physics, in recognition of recent work (i.e. within the past five years).

Proposals are now invited for the 1984 award. These may be submitted by Members individually, or as representatives of a Division and should include:

a clear definition of the work in question; a short biography of the candidate;

a list of relevant publications and reprints referring to the work recommended.

Details should be sent to the Selection Committee, c/o EPS Secretariat, POB 69, $\mathrm{CH}-1213$ Petit-Lancy 2, to arrive not later than 12 August 1983. All information will be treated as confidential.
EPS Divisions, Sections and Group Astronomy and Astrophysics Division

Solar Physics Section
Atomic and Molecular Physics Division Atomic and Molecular Physics Divis
Atomic Spectroscopy Section Chemical Physics Electronic and Atomic Collisions Molecular Physics Computational Physics Group Condensed Matter Division Low Temperature Physics Section Macromolecular Physics Magnetism Metal Physics Semiconductors and Insulators Surfaces and Interfaces High Energy \& Particle Physics Division Nuclear Physics Division Optics Division Optics Division
Plasma Physics Division Plasma Physics Division
Europhysics News is the official journal of the European Physical Society which comprises 29 National Societies. Academies and Groups, over 3000 Individual Members and 62 Associate Members. Governing bodies of EPS are the General Meeting. Council and an elected Executive Committee responsible for detailed policy. EPS promotes the collaboration of physicists throughout Europe, organising and harmonising conferences, and promotes international exchanges in phy. sics including participation in research and teaching activities abroad and attendance at schools. EPS publishes in addition to EN, Europhysics Conference Abstracts, E. Ed. News and, in collaboration with The Institute of Physics (UK), the European Journal of Institute of Physics (UK), the European Journal of
Physics. Individual Members receive EN free of charge (price to institutions: Sw.Fr. 82. - /a), rebates on the price of many publications and on conference fees. An nual EPS membership fee for Individual Members who belong to one of the EPS member societies for 1983 is: Sw.Fr. 40.-; for independent members: Sw. Fr. 120.-
Editor: E.N. Shaw Meetings Compilation: W.S. Newman Editorial Board

K. Appert, A. Baratoff, G.J. Béné,

G.R. Macleod, A. Maeder, J. Muller

Editorial and Advertising Office at the EPS Secretariat.

Address: EUROPEAN PHYSICAL SOCIETY P. O. Box 69 . $\mathrm{CH}-1213$ Petit-Lancy 2 Switzerland

Telephone: Geneva (22) 931130

Telex: 23455 alarm ch

Cables: europhys genève

Printed by: Pfirter frères SA $\mathrm{CH}-1213$ Petit-Lancy/Switzerland 